\title{
End-of-Life Care
}

\section{Roland Kunz}

Dr. med, Chefarzt universitäre Klinik für Akutgeriatrie und Zentrum für Palliative Care Stadtspital Waid, Zürich

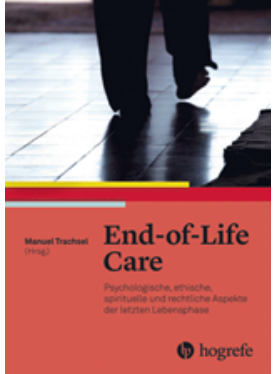

Manuel Trachsel (Hrsg.)

End-of-Life Care

Psychologische, ethische, spirituelle und rechtliche

Aspekte der letzten Lebensphase

Bern: Hogrefe; 2018. 152 Seiten.

ISBN 978-3-456-85858-6

In den letzten Jahren haben die Neuerscheinungen zum Thema Palliative Care zahlenmässig einen eigentlichen Boom erlebt. Am Anfang war der Wissenszuwachs im immer noch jungen Fachgebiet gross, mit jeder neuen Publikation nahm er aber zwangsläufig ab. Die Autoren versuchten zunehmend, ihre neuen Bücher mit der Fokussierung auf Teilaspekte der Thematik besser zu platzieren.

Nun präsentiert uns Manuel Trachsel als Herausgeber ein Buch zum Thema End-of-Life Care. Gespannt las ich die Beiträge der verschiedenen Autoren mit der Frage, wieweit sich zur allerletzten Lebensphase noch mehr sagen lässt, als schon bekannt ist aus der bisherigen Literatur zu Palliative Care. Um es vorwegzunehmen: Nicht alle Kapitel werden den Erwartungen gerecht. Das vom Herausgeber verfasste Kapitel «Psychische Belastungsfaktoren im Sterbeprozess» fasst Bekanntes zur Symptomkontrolle zusammen. Diese Zusammenfassung bleibt aber weitgehend auf einem allgemeinen Informationsniveau und nimmt kaum Stellung zu den besonderen Herausforderungen am Lebensende in der Praxis. Wie schwierig die Abgrenzung der End-of-Life Care ist, zeigt sich exemplarisch beim Unterkapitel «Schmerzen im Sterbeprozess». Wenn hier über die allgemeine Schmerzphänomenologie und über die Chronifizierung von Schmerzen geschrieben wird, bleibt der Bezug zum Fokus des Buches weitgehend aus. Dass es eben kaum Neues zu berichten gibt, zeigt sich auch daran, dass das Kapitel mit einer Flut von Zitaten aus früheren Publikationen des Autors gespickt ist.

Sehr lesenswert sind die Ausführungen von Ralf Jox zu den ethischen Herausforderungen am Lebensende.
Fragen der Therapiebegrenzung, der Entscheidungsfindung, des freiwilligen Verzichts auf Essen und Trinken oder der palliativen Sedierung sind konkret auf die End-of-Life Care bezogen und werden mit Blick auf die Praxis vertieft und hilfreich diskutiert. Auch das Kapitel «Spiritual Care» von Hubert Kössler und Thomas Wild verbindet grundsätzliche Überlegungen zum oft schwer fassbaren Inhalt verständlich mit praxisbezogenen Empfehlungen zur Integration in die Begleitung Sterbender.

Im Abschnitt zu den rechtlichen Aspekten wünschte man sich nicht nur eine Abhandlung über juristische Fakten, sondern auch eine Wertung oder Diskussion der Gesetzestexte. Zum Beispiel der Begriff der indirekten (aktiven) Sterbehilfe ist ein juristisches Konstrukt, das in der Praxis kaum Relevanz hat und eher kontraproduktiv ist, weil es Ärzte von einer suffizienten Schmerzbehandlung abhalten kann. Eine entsprechende Gewichtung wäre hilfreich, ebenso ein Bezug der Rechtslage der Suizidbeihilfe zu den aktuellen gesellschaftlichen Diskussionen. Das letzte Kapitel befasst sich mit der klinischen Ethik bezogen auf die End-of-Life Care. Auch dieser Teil des Buches bleibt sehr allgemein, stellt Konzepte der Ethikberatung vor, beschreibt ein Fallbeispiel zum Thema Schwangerschaftsabbruch, lässt aber die zentralen Themen schwieriger Entscheidungen am Lebensende wie die palliative Sedation oder den Suizidwunsch unbesprochen.

Vielleicht habe ich zu viel erwartet unter dem Titel des Buches. Es bleibt auch nach dem Lesen unklar, was das Ziel und das Zielpublikum des Buches sind. Für Mitarbeitende aus dem Bereich Palliative Care bietet es wenig Neues, für Einsteiger ins Thema ist es zu wenig praxisbezogen. Aber trotzdem habe ich aus einigen Kapiteln wie beschrieben neue Impulse erhalten. Das Buch kann deshalb mehrheitlich zum punktuellen Einsatz als orientierendes (theoretisches) Nachschlagewerk empfohlen werden, andererseits können die Kapitel zu den ethischen Herausforderungen und zu den spirituellen und religiösen Aspekten auch in Palliative Care erfahrenen Ärzten neue Impulse geben. 